\title{
Worth holding on to
}

Most natural killer T (NKT) cells express an invariant $\mathrm{T}$ cell receptor (TCR) and are collectively known as invariant NKT (iNKT) cells. The invariant TCRs of mammalian iNKT cells recognize a highly conserved set of antigens (including self antigens and bacterial glycolipids) that are presented on MHC class I-like CD1d molecules. However, it has not been clear why this TCR specificity has persisted throughout evolution. Now, a recent study in Nature Immunology has shown that the TCR of iNKT cells directly recognizes glycolipids expressed by highly pathogenic Streptococcus spp., thereby offering an explanation for its conservation.

Although previous work had described a protective role for iNKT cells during Streptococcus pneumoniae infection in mice, the mechanisms of this were not known and it was unclear whether foreign antigen recognition was required. Kinjo et al. found that interferon- $\gamma$ $(\mathrm{IFN} \gamma)$-producing iNKT cells could be isolated from the lungs of mice infected with S. pneumoniae at early time points following intratracheal infection.

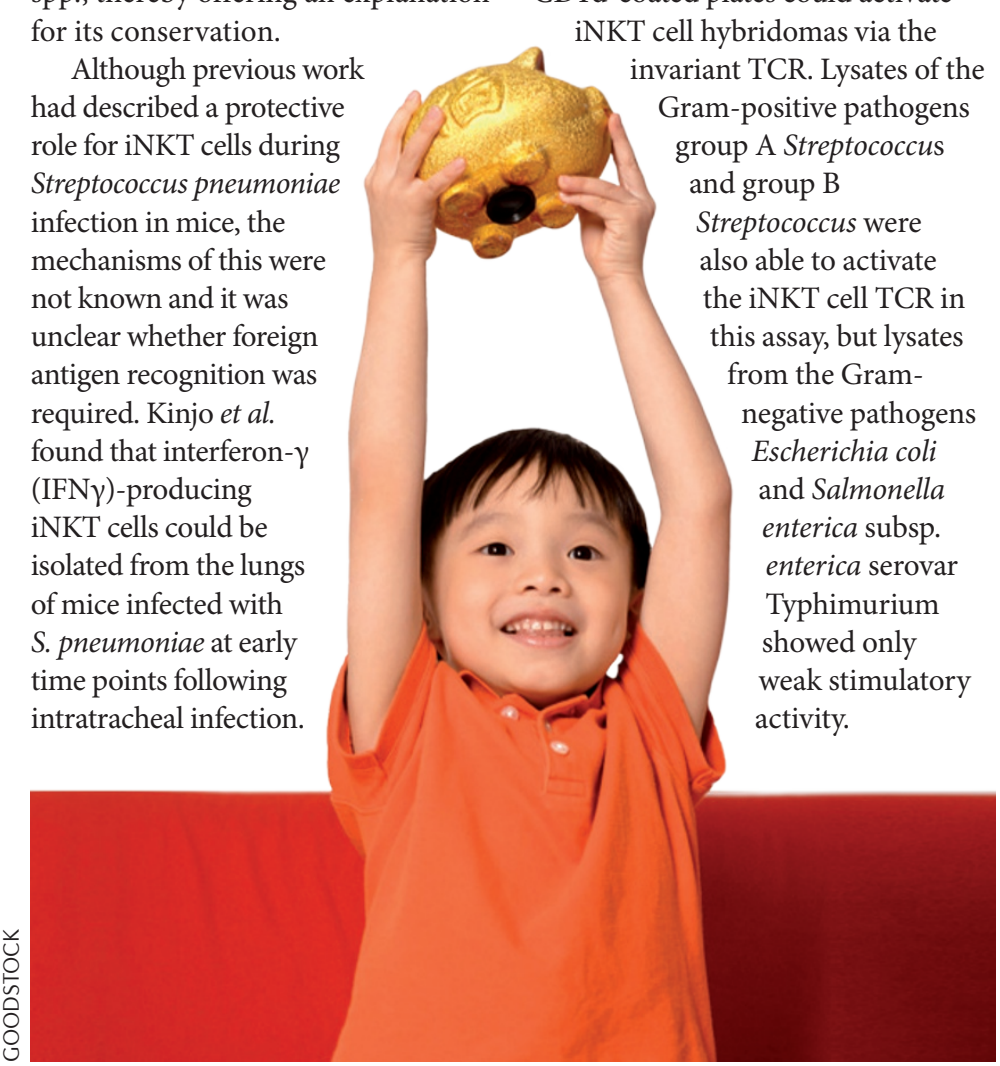

However, when infected mice were treated with blocking antibodies specific for CD1d, decreased frequencies of IFN $\gamma$-producing iNKT cells were found and increased bacterial loads occurred. This suggested that iNKT cells were activated directly through their invariant TCR during S. pnemoniae infection and contributed to bacterial clearance.

The authors next explored whether iNKT cells are activated by self or microbial antigens during S. pneumoniae infection. Using an in vitro model to assess iNKT cell TCR activity, they showed that S. pneumoniae lysates added to CD1d-coated plates could activate iNKT cell hybridomas via the variant TCR. Lysates of the also able to activat the iNKT cell TCR in ysates from the Gramnegative pathogens Escherichia coli and Salmonella enterica subsp. enterica serovar Typhimurium showed only weak stimulatory
Structural analyses identified two main fractions of $S$. pneumoniae glycolipids, one with a single glucose molecule $\alpha$-linked to diacylglycerol and another with a glucose-galactose disaccharide moiety $a$-linked to diacylglycerol. Both glycolipid fractions could activate iNKT cell hybridomas via the invariant TCR, and experiments using synthetic ligands showed that this depended on the presence of vaccenic acid, a fatty acid that is not commonly found in mammalian cells. Transfer of bone marrow-derived dendritic cells that had been pulsed with the S. pneumoniae glycolipids led to NKT cell activation in mice, showing that these bacterial glycolipids can also induce NKT cell responses in vivo. Importantly, this occurred even when the transferred glycolipidpulsed DCs were defective in Toll-like receptor (TLR) signalling or unable to produce interleukin-12, suggesting that NKT cell activation depends on invariant TCR stimulation rather than on TLR-mediated innate immune responses.

The authors suggest that the unique glycolipids that they have identified in S. pneumoniae might represent a new type of pathogenassociated molecular pattern that is recognized by the invariant TCR of iNKT cells. They propose that iNKT cells may have been conserved throughout evolution owing to their ability to recognize pathogens that express these glycolipids.

Yvonne Bordon

ORIGINAL RESEARCH PAPER Kinjo, Y. et a

Invariant natural killer T cells recognize glycolipids from pathogenic Gram-positive bacteria. Nature Immunol. 4 Sep 2011 (doi:10.1038/ni.2096) 